\title{
A Case of a Giant Growing Serous Cystic Neoplasm of the Pancreas
}

\author{
Yoshiaki Kawaguchi $^{\mathrm{a}} \quad$ Takayuki Nakajima $^{\mathrm{a}}$ Yohei Kawashima $^{\mathrm{a}}$ \\ Atsuko Maruno ${ }^{a}$ Hiroyuki Ito $^{a}$ Masami Ogawa $^{a}$ Tetsuya Mine ${ }^{a}$ \\ Hideki Izumi $^{\mathrm{b}}$ Daisuke Furukawa ${ }^{\mathrm{b}}$ Naoki Yazawa ${ }^{\mathrm{b}}$ Toshio Nakagohri ${ }^{\mathrm{b}}$ \\ Kenichi Hirabayashic \\ Departments of ${ }^{\mathrm{a}}$ Gastroenterology, ${ }^{\mathrm{b}}$ Surgery and ${ }^{\mathrm{c}}$ Pathology, Tokai University School of \\ Medicine, Isehara, Japan
}

\section{Key Words}

Serous cystic neoplasm · Pancreas · Giant size · Diagnosis

\begin{abstract}
Because of the widespread use of diagnostic imaging in recent years, serous cystic neoplasm (SCN) of the pancreas can often be detected even when small in diameter. SCNs are usually benign, but it is important to differentiate them from other types of cystic tumors. We report a case of a giant growing $\mathrm{SCN}$ that posed a challenge in differential diagnosis.
\end{abstract}

(C) 2014 S. Karger AG, Basel

\section{Case Report}

In 2003, during a routine health checkup, a pancreatic cyst measuring $8 \mathrm{~cm}$ in diameter was detected in a 51-year-old woman. The cyst was subsequently managed with follow-up care by the previous physician on an outpatient basis. In 2010, the cyst had increased to 12 $\mathrm{cm}$ in size and then to $19 \mathrm{~cm}$ within approximately the next 6 months. At this time, the patient was referred to our hospital for treatment. At the initial visit, she reported abdominal bloating. However, no medical problems were evident from the patient's health history, and she had no history of pancreatitis, abdominal trauma or alcohol intake. A physical examination showed no sign of anemia in the palpebral conjunctiva or jaundice of the bulbar conjunctiva; the abdomen was soft, flat, and nontender, and no obvious tumor mass was detected by palpation. Blood tests revealed no elevation of pancreatic enzyme levels or tumor markers. Abdominal ultrasonography and contrast-enhanced abdominal computed 
tomography (CT) scans showed the presence of a partially septated giant cystic lesion measuring $20 \times 11 \times 13 \mathrm{~cm}$ in the abdominal cavity (fig. 1). The lining membrane of the cyst was thin, and no solid components or calcifications were noted in the cyst. A beak sign was observed in the pancreas, and the main pancreatic duct in the tail of the pancreas was slightly dilated. Therefore, the mass was considered a cystic tumor of pancreatic origin. On abdominal magnetic resonance imaging, the pancreatic body lesion detected by the CT scan was depicted as high-intensity area on T2-weighted images. These findings were consistent with the characteristics of cystic lesions (fig. 2). A septum-like structure was present in the cyst, and dilation was noted in the pancreatic duct in the tail of the pancreas. Endoscopic ultrasonography showed extramural compression of the middle part of the gastric body. Although imaging studies of the same site could not reveal the entire extent of the cyst because of its enormous size, the cyst demonstrated low-level internal echoes and did not show any obvious solid component. A septum-like structure was observed, but there was no microcystic lesion with a characteristic honeycomb pattern. Endoscopic retrograde cholangiopancreatography displayed only the main pancreatic duct in the head of the pancreas; the cyst compressed the tail side. Based on these findings, the differential diagnosis included serous cystic neoplasm (SCN), mucinous cystic neoplasm, and branch duct-type intraductal papillary mucinous neoplasm in the neoplastic category as well as pancreatic pseudocyst in the nonneoplastic category. Because this mass was increasing in size and abdominal bloating was present, surgical resection was deemed appropriate. Therefore, a distal pancreatectomy with splenectomy was performed. The cystic content was serous fluid of a dark, muddy color, suggesting previous bleeding (fig. 3). On histopathological examination, the lining membrane had a papillary structure composed of cuboidal cells with round nuclei. The cells stained PAS-positive and diastase PAS-negative, thus indicating the presence of glycogen (fig. 4). These findings led to the definitive diagnosis of SCN.

\section{Discussion}

An SCN is a relatively rare type of tumor characterized by a cluster of multiple small cysts arranged in a honeycomb formation that develops more commonly in middle-aged and older women. An SCN can occur at any site in the pancreas, but is more frequent in the pancreatic body and tail $[1,2]$. With the advances in diagnostic imaging technology in recent years, the detection rates of SCN are believed to be on the increase. On histological examination, each cyst is lined with a flat-to-cuboidal epithelium and consists of glycogen-rich clear cells. SCN is a multilocular cystic tumor characterized by multiple small cysts clustered in a honeycomb appearance. Many small blood vessels are located immediately beneath the epithelium (subepithelial capillary network), and the stroma is a proliferation of numerous capillaries and fibrous tissues. Very few cases of malignant SCN have been reported thus far, but cases of liver metastasis, invasion, and recurrence have been reported, some of which had malignant potential. Therefore, careful examination of the mass is required [3-8].

Most small SCNs are asymptomatic. However, as the mass increases in size, subjective symptoms such as bloating and epigastric pain appear; there are no specific symptoms. In the present case, the patient experienced abdominal bloating as the lesion increased in size.

Central calcifications are observed in imaging studies in $10-20 \%$ of the cases, and contrast-enhanced CT scans show the septum and connective tissue as areas of high density. Depending on the size of the septum and connective tissue, the lesion may have a sponge or net-like-to-solid appearance [1]. On magnetic resonance imaging, the lesion exhibits a marked high-intensity signal on T2-weighted images in which the sponge-like or honeycomb 
structure is clearly visible. This is considered a characteristic feature of SCN and is thus useful for definitive diagnosis [1]. In the present case, a beak sign was noted in the pancreas, with dilation of the main pancreatic duct in the pancreatic tail. This finding was suggestive of a cystic tumor of pancreatic origin. However, the images showed no calcification, very few septal structures, and no evidence of the honeycomb pattern unique to SCN. It is especially difficult to distinguish between a macrocystic type of a tumor like the lesion described and an MCN, posing a challenge to differential diagnosis.

Most SCN cases are benign and generally managed with follow-up care alone. However, surgical resection is performed if the lesion is large, increases in size, or causes symptoms. If an SCN grows to a giant size, it can compress or invade surrounding organs or vascular channels. In fact, studies have reported cases of obstructive jaundice caused by compression of the bile duct and portal hypertension caused by compression of the portal vein [9-14]. Although the lesion in the present case was large, it did not have a notable influence on the surrounding organs or tissues. However, the outcome could have been different if the detection of the lesion had been delayed. Malignant cases have been reported with SCNs measuring $>6 \mathrm{~cm}$ in diameter, and some researchers recommend active resection for SCNs $>4 \mathrm{~cm}$ in diameter. The lesion in the present case demonstrated growth during the follow-up period; however, no malignant findings were observed on pathological examination. As the cyst contained dark, muddy, serous fluid that suggested previous bleeding, the observed growth was thought to have resulted from bleeding within the cyst.

As SCNs are usually benign, it is important to differentiate them from other types of cystic tumors. This case was a rare giant growing SCN that posed a challenge in differential diagnosis.

\section{References}

$\checkmark 1$ Compagno J, Oertel JE: Microcystic adenomas of the pancreas (glycogen-rich cystadenomas). A clinicopathologic study of 34 cases. Am J Clin Pathol 1978;69:289-298.

-2 Morohoshi T, Held G, Kloppel G: Exocrine pancreatic tumours and their histological classification. A study based on 167 autopsy and 97 surgical cases. Histopathology 1983;7:645-661.

-3 Kamei K, Funabiki T, Ochiai M, Amano H, Kasahara M, Sakamoto T: Multifocal pancreatic serous cystadenoma with atypical cells and focal perineural invasion. Int J Pancreatol 1992;10:161-172.

-4 Abe H, Kubota K, Mori M, Miki K, Minagawa M, Noie T, et al: Serous cystadenoma of the pancreas with invasive growth: benign or malignant? Am J Gastroenterol 1998;93:1963-1966.

5 Widmaier U, Mattfeld T, Siech M, Berger HG: Serous cystadenocarcinoma of the pancreas. Int J Pancreatol 1996;20:135-138.

6 Eriguchi N, Aoyagi S, Nakayama T: Serous cystadenoma of the pancreas with liver metastasis. J Hepatobiliary Pancreat Surg 1998;5:467-470.

7 Ohta T, Nakakawa T, Itoh H, Fonseca L, Miyazaki I, Terada T: A case of serous cystadenoma of the pancreas with focal malignant changes. Int J Pancreatol 1993;14:283-289.

8 Formentini A, Birk D, Siech M: Serous cystadenocarcinoma of the pancreas and serous cystadenoma associated with ductal pancreatic adenocarcinoma. HPB Surg 2000;2:41-45.

-9 Maekawa T, Yabuki K, Satoh K, Tamasaki Y, Amano T, Sengoku H, et al: A case of pancreatic serous cystadenoma with obstructive jaundice. Dig Endosc 2000;12:55-60.

10 Watanabe H, Ohtsubo K, Yamaguchi Y, Mouri H, Motoo Y, Noto M, Kitagawa H, Kayahara M, Ohta T, Gabata T: Successful cystic fenestration for a macrocystic serous cystadenoma of the pancreas causing obstructive jaundice: report of a case. Surg Today 2006;36:89-93.

$\rightarrow 11$ Horaguchi J, Fujita N, Kobayashi G, Noda Y, Kimura K, Ito K, et al: Serous cystadenoma of the pancreas associated with obstructive jaundice. J Gastroenterol 2003;38:501-506.

$\rightarrow 12$ Okuyama M, Fujiwara Y, Hayakawa T, Shibata M, Watanabe T, Tominaga K, et al: Esophagogastric varices due to arterioportal shunt in a serous cystadenoma of the pancreas in von Hippel-Lindau disease. Dig Dis Sci 2003;48:1948-1954.

13 Iwasaki T, Nagata Y, Watahiki H, Yamamoto H, Ogawa H: A rare case of serous cystadenoma of the pancreas presenting with left-sided portal hypertension. Surg Today 1996;26:442-445.

14 Ito K, Kudo A, Nakamura N, Tanaka S, Teramoto K, Arii S: Left-sided portal hypertension caused by serous cystadenoma of the pancreas: report of a case. Surg Today 2008;38:184-187. 


\section{Case Reports in Oncology}

Case Rep Oncol 2014;7:47-51
DOI: $10.1159 / 000358048$

Kawaguchi et al.: A Case of a Giant Growing Serous Cystic Neoplasm of the Pancreas

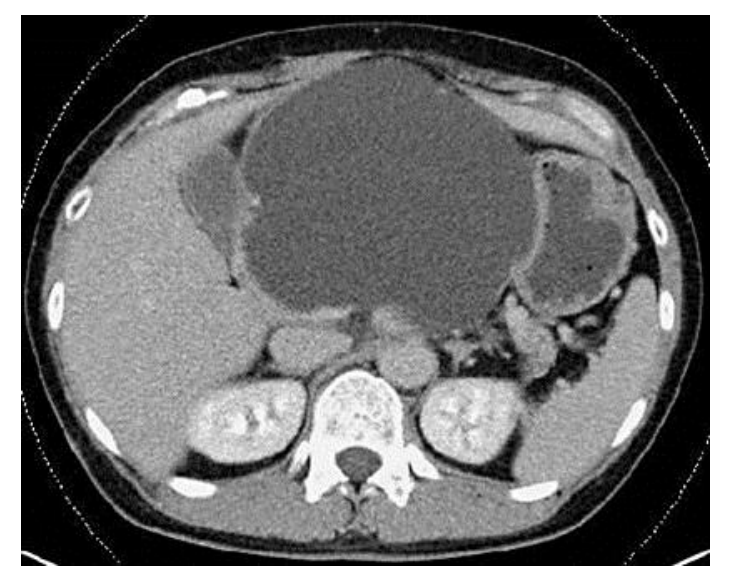

Fig. 1. Contrast-enhanced abdominal CT shows the presence of a partially septated giant cystic lesion measuring $20 \times 11 \times 13 \mathrm{~cm}$ in the abdominal cavity.

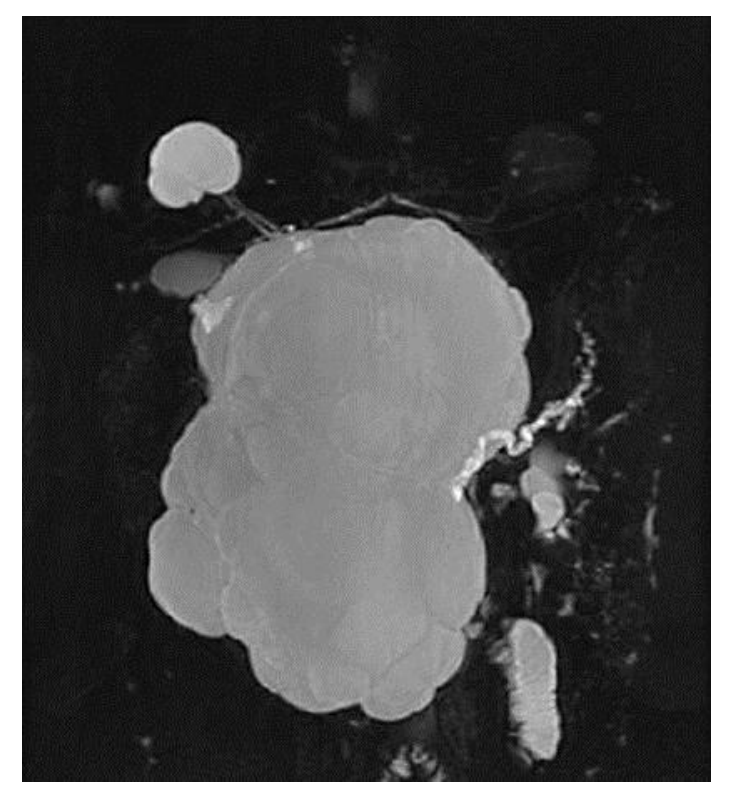

Fig. 2. On magnetic resonance cholangiopancreatography, the pancreatic body lesion detected by the CT scan was depicted as high-intensity area on a T2-weighted image. 


\section{Case Reports in Oncology}

\begin{tabular}{l|l}
\hline Case Rep Oncol 2014;7:47-51 \\
\hline DOI: $10.1159 / 000358048$ & $\begin{array}{l}\text { (c) 2014 S. Karger AG, Basel } \\
\text { www.karger.com/cro }\end{array}$ \\
\hline
\end{tabular}

Kawaguchi et al.: A Case of a Giant Growing Serous Cystic Neoplasm of the Pancreas

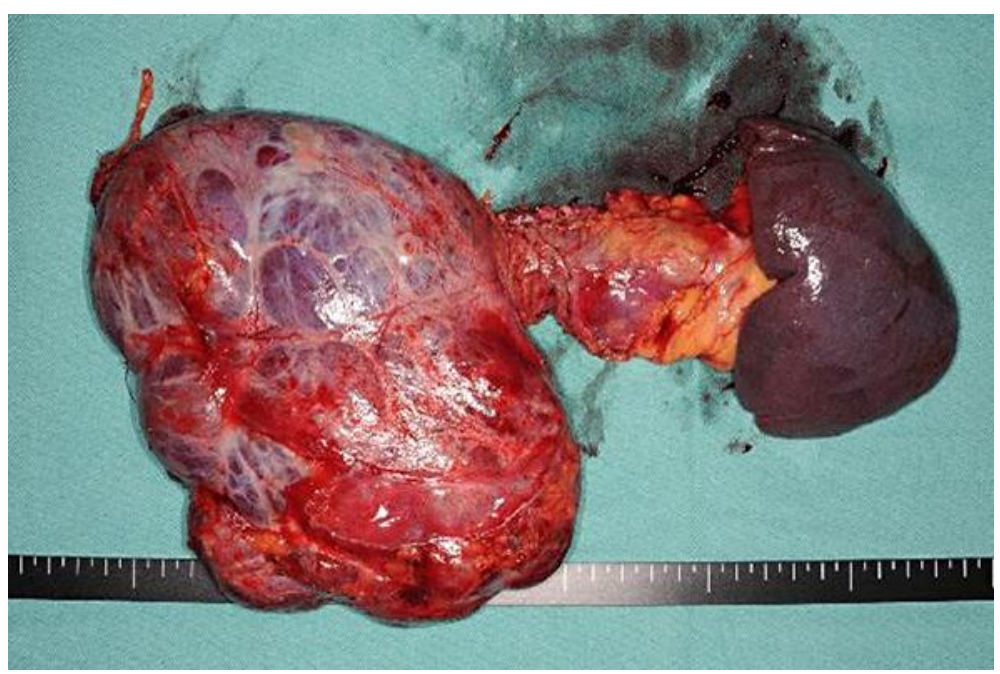

Fig. 3. A distal pancreatectomy with splenectomy was performed. The cystic content was serous fluid of a dark, muddy color, suggesting previous bleeding.
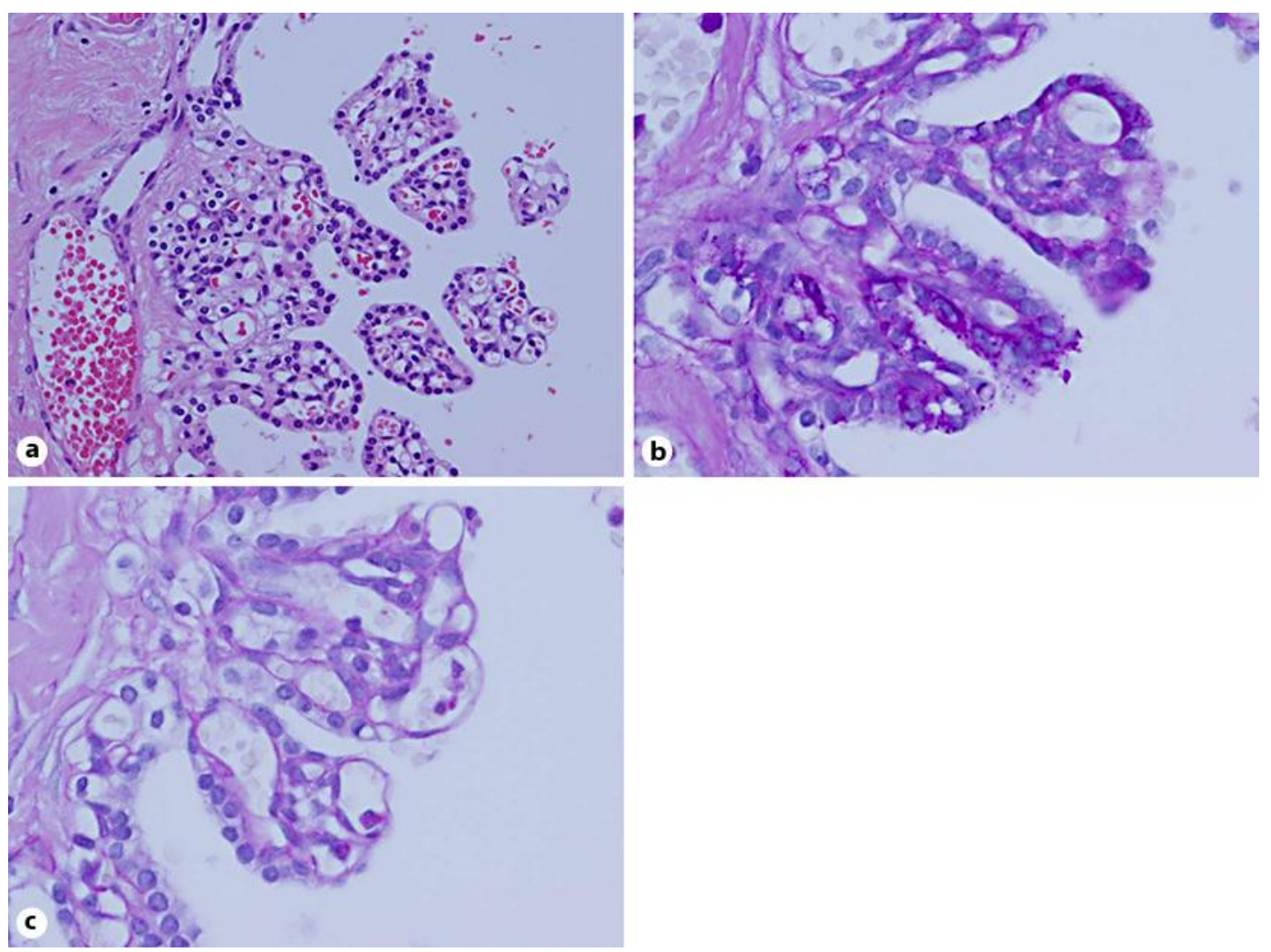

Fig. 4. On histopathological examination, the lining membrane had a papillary structure composed of cuboidal cells with round nuclei (a). The cells stained PAS-positive (b) and diastase PAS-negative (c), thus indicating the presence of glycogen. These findings led to the definitive diagnosis of SCN. 